\title{
Analysis of metastatic bone disease in a tertiary care centre
}

\author{
Subalakshmi .B ${ }^{1}$, B. Nellaiyappan ${ }^{2, *}$, J. Thanka ${ }^{3}$ \\ ${ }^{1,2}$ Assistant Professor, ${ }^{3}$ Professor, ${ }^{1,3}$ Dept. of Pathology, ${ }^{2}$ Dept. of Orthopaedics, Sri Ramachandra Medical College and Research \\ Institute, (Sri Ramachandra University), Chennai, Tamil Nadu, India
}

*Corresponding Author:

Email: nellaisuba12@gmail.com

Received: $20^{\text {th }}$ March, 2018

Accepted: $9^{\text {th }}$ April, 2018

\begin{abstract}
Introduction: Metastasis to the bone is the third commonest site next to lung and liver. It is one of the leading cause of morbidity and mortality in the world being, an important prognostic indicator in advanced cancers. The axial skeleton is more commonly involved than the appendicular skeleton due to the venous blood flow through the bat sons plexus. Commonest site involved are the spine, femur, pelvis, skull.

Materials and Methods: A retrospective analysis of cases with metastatic bone disease confirmed by histopathology and followed by immunohistochemistry was carried out in the department of Pathology, SRMC from January 2014 to December 2016. The data of same were analyzed and recorded.

Results: A total of 36 cases with bone metastasis were analyzed of which 26 patients were males and 10 were females. Metastasis from epithelial tumors were $94 \%$ as compared to $6 \%$ of deposits from mesenchymal tumor. Most common site of involvement was the spine (50\%) second most common being femur (25\%) followed by the iliac bone (14\%) and clavicle (3\%), tibia (3\%), skull (3\%), radius (3\%) in both males and females. Most common primary site of origin were the Lung (29\%), followed by prostate (17\%), Gastrointestinal tract (14\%), renal (11\%), breast $(6 \%)$, thyroid (6\%), ovary (3\%) penis $(3 \%)$ liver (3\%), Thigh - soft tissue (3\%) and uterus (3\%). For 3 cases $(8 \%)$ the primary site was unknown.

Conclusion: Patients presenting with metastatic bone disease is increasing over time but advancement in treatment and awareness among patients have helped in early detection and prompt treatment and longer survival.
\end{abstract}

Keywords: Metastasis, Bone, Primary, Immunohistochemistry.

\section{Introduction}

Metastatic bone disease is one of the commonest malignancies involving the bone and a leading cause of morbidity and mortality as it portends a decreased survival and poor outcome. Prostate, kidney, breast, lung and thyroid carcinomas account for $80 \%$ of skeletal metastasis. ${ }^{1}$ The commonest site of bone metastasis are the spine, pelvis, proximal femur, skull, ribs and rarely to the distal metastasis to the hand and feet. Advanced studies show that specific bones have an affinity for metastatic deposits and it is regulated by cytokines, chemokines and other growth factors which is present in the microenvironment of the bone. Patients are usually diagnosed during staging or by Tech. bone scan. The clinical presentation is variable depending on the location. Most common symptoms being pain, followed by pathological fracture and neurological deficit. Involvement of weight bearing bones present with earlier manifestations where as involvement of the flat bones are asymptomatic until late in the course of the disease. Imaging studies play a role in determining the nature of the disease. ${ }^{2}$ Plain radiograph helps us to assess the initial nature of the disease, Computed Tomography, Magnetic Resonance Imaging is useful to know the extent of the disease involved. By radiographic appearance bone lesion are classified as osteoblastic, osteolytic or mixed. Metastasis from bladder, prostate, medulloblastoma are most likely to be osteoblastic in nature. Whereas osteolytic metastasis are seen with renal, thyroid, gastrointestinal tract and adrenal malignancies. Mixed type are seen with Breast and ovary. ${ }^{3}$

Morphology of the metastatic lesion to the bone is non specific. Grossly it may be grey white with a consistency which may be firm with solid tumors like breast and cystic or hemorrhagic with renal cell carcinoma and papillary carcinoma. ${ }^{4}$ By microscopy metastatic tumors resemble the primary tumor of origin. In cases where the histology is undifferentiated or poorly differentiated tumor immunohistochemistry plays an important role in determining the primary site of the tumor. The immunohistochemical evaluation of a poorly differentiated neoplasm should first aim in sub categorization of the tumor as epithelial, sarcomatoid or of lymphoid origin. Variety of screening markers are available to sub categorize as epithelial/ mesenchymal for example Immunohistochemistry (IHC) for EMA /CK are positive in epithelial tumors. CD45 is positive in tumors of lymphoid origin. SMA, S100, Calretinin are positive in tumors of mesenchymalorigin. ${ }^{5}$

\section{Materials and Methods}

Aim of the study is to analyses the frequency and distribution of metastatic bone disease in this part of our country. This is a retrospective analysis of data carried out in the department of Pathology, SRMC from January 2014 to December 2016. Thirty six cases presented with bone metastasis for which 
Hematoxylin\& Eosin diagnosis was initially interpreted followed by IHC. Data was analyzed and recorded.

\section{Observation and Results}

Total of 36 cases of bone metastasis were analyzed of which 26 were male patients and 10 were females. Age of incidence of the patients ranged between 40-75 years with higher incidence seen in the age group of 50 - 60 years. Metastasis from epithelial tumors $(94 \%)$ were more common than the mesenchymal tumor $(6 \%)$. Epithelial tumors included adenocarcinoma from lung, prostate, colon and renal cell carcinoma \& follicular carcinoma thyroid. Mesenchymal tumors included myxoid fibrosarcoma and leiomyosarcoma. Most common site of bones involved was the spine which included 19 cases (53\%) second most common being femur which was seen in 8 cases $(25 \%)$ followed by the iliac bone in 5 of them (14\%) and the least involved were the clavicle $(3 \%)$, tibia (3\%), skull $(3 \%)$, radius $(3 \%)$ in both males and females. Most common primary site of origin to metastasise were from the lung (29\%), followed by prostate $(17 \%)$, G.I.T (14\%), renal $(11 \%)$, beast $(6 \%)$, thyroid $(6 \%)$, ovary $(3 \%)$ penis $(3 \%)$, liver $(3 \%)$, thigh soft tissue $(3 \%)$ uterus $(3 \%)$. For 3 cases (8\%) the primary site was unknown. A panel of immunohistochemistry markers were done to confirm the origin of tumor in all the cases. The commonest tumor in our study were from the lung for which IHC CK7 and CK 20 and TTF - 1 was done which took up cytoplasmic positivity for CK 7 and nuclear positivity for TTF 1 and negative for CK 20. For prostatic adenocarcinoma nuclear positivity was taken up PSA. For colonic tumors CK 7, CK 20 and CDX 2 was done which showed cytoplasmic positivity for Cytokeratin 20 and CDX2 and negative for CK7. A panel of epithelial markers CK7, CK 20, CDX2, \& EMA was done to rule out primary from the stomach for which cytoplasmic positivity was taken by EMA and negative for CK7, CK 20 and CDX2. For tumors from renal origin CD10 was positive in the membrane of the tumor cells. For breast carcinomas ER, PR was done which took nuclear positivity. TTF-1 was positive in the thyroid carcinomas which took up nuclear positivity. Metastasis from ovarian serous carcinoma showed CK 7, EMA, WT 1 positivity and CK 20 Negative. For metastasis from liver Glypican and Hepar-1 was positive which took up cytoplasmic positivity in the tumor cells. Squamous cell carcinoma from penis was seen to infiltrate the bone by morphology the diagnosis was given and P63 was done for confirmation which was nuclear positive. For mesenchymal tumors one case of leiomyosarcoma was seen infiltrating the bone for which SMA was positive in the tumor cells and for fibrosarcoma all the markers of soft tissue tumors (EMA, S100, SMA, CD34) were negative and correlating with $\mathrm{H} \& \mathrm{E}$ diagnosis and imaging studies the diagnosis was given.

\section{Graph 1}

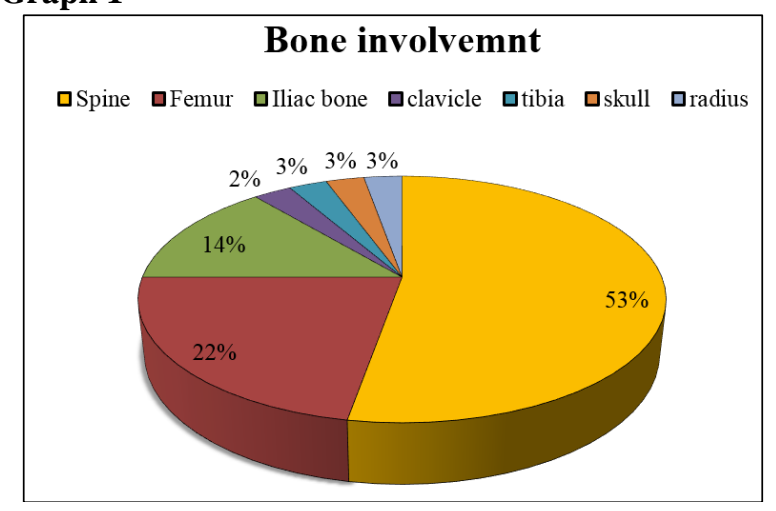

\section{Graph 2}
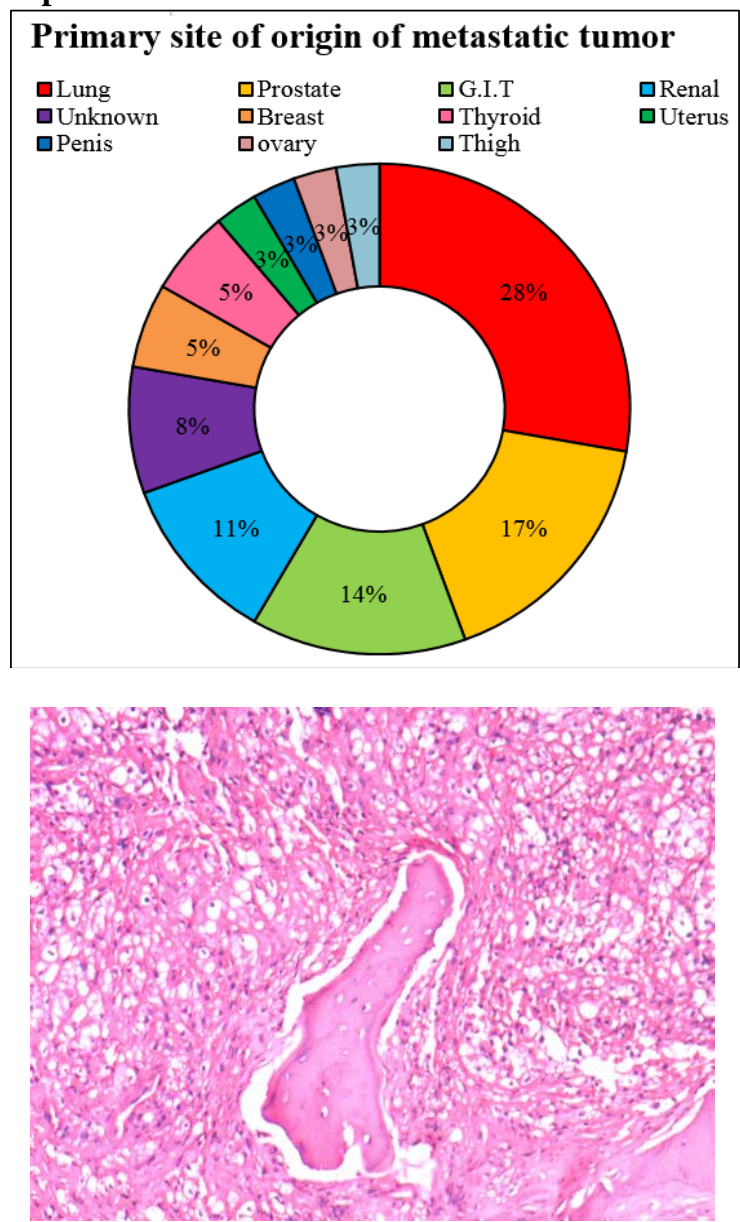

Fig. 1: H\&E 200x metastatic deposit from clear cell renal cell carcinoma 


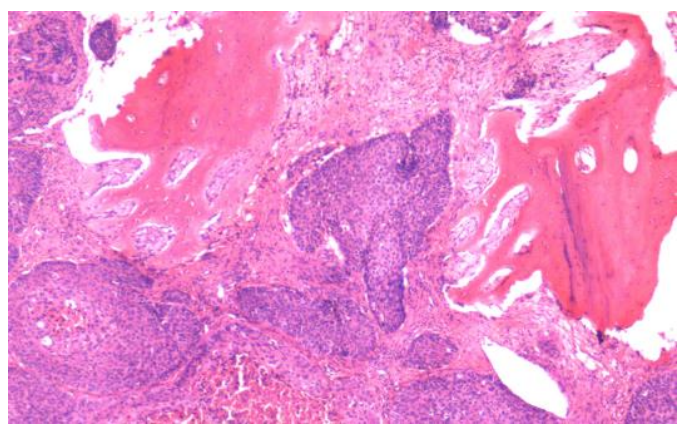

Fig. 2: H \& E 200X metastatic deposit from squamous cell carcinoma

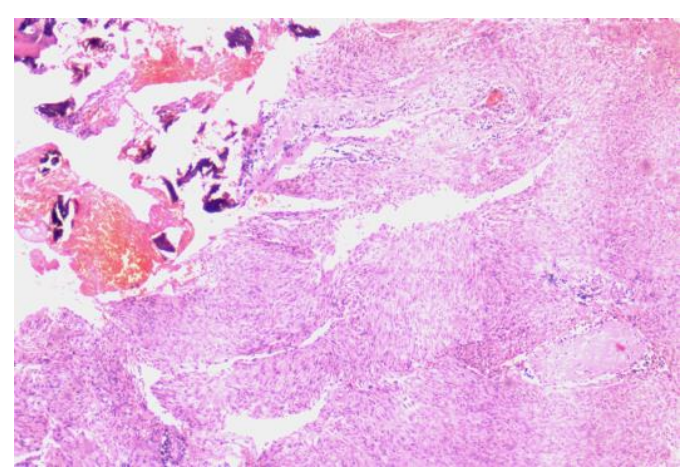

Fig. 3: H \& E 200x metastatic deposit of leiomyosarcoma to the iliac bone

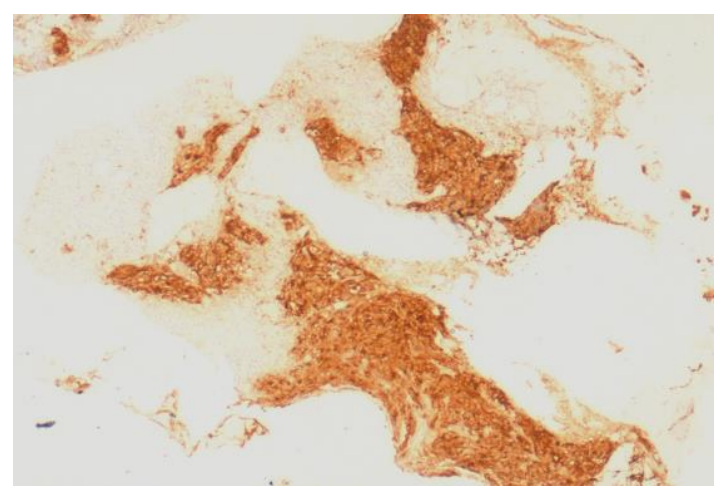

Fig. 4: IHC 200X PSA positivity in the tumor cells from metastatic deposit from prostatic adenocarcinoma

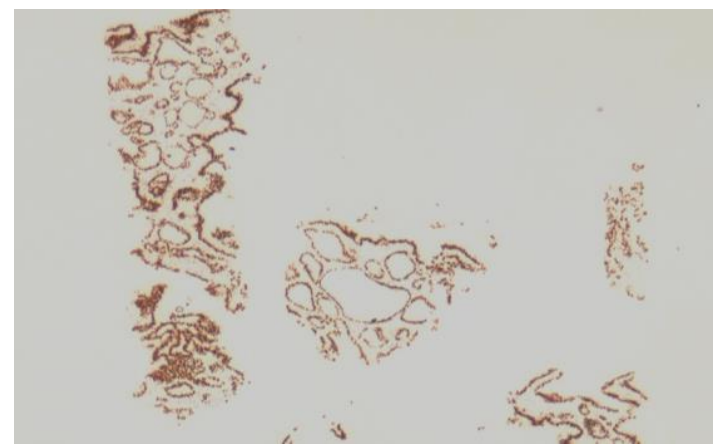

Fig. 5: IHC 200X TTF-1 shows positivity in the tumor cells from metastatic deposit of follicular variant of papillary carcinoma thyroid.

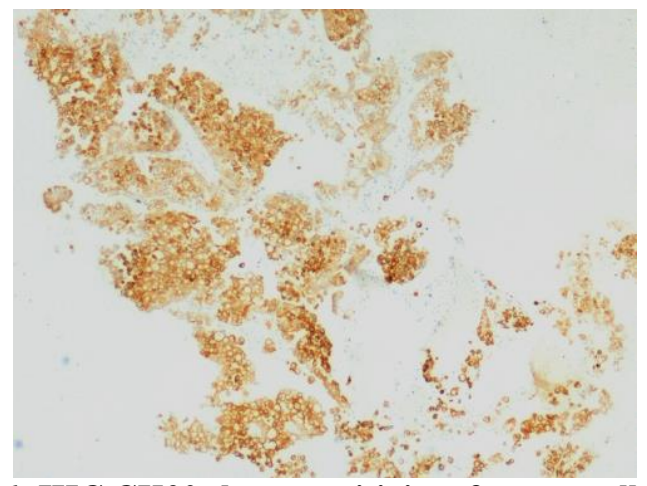

Fig. 6: IHC CK20 shows positivity of tumor cells from metastatic signet ring cell carcinoma from rectum

\section{Discussion}

Metastatic bone disease is a debilitating disease with an increased morbidity and is associated with a poor outcome. Involvement of a specific bone depends on the anatomic location and vascular supply of the primary site. In our study more than $60 \%$ of the patient presented in the $6^{\text {th }}$ to $7^{\text {th }}$ decade of life, average ranging from 40 to 60 years of which there were 26 males and 10 where females, David et al found that the commonest age group was between $3^{\text {rd }}$ and $4^{\text {th }}$ decade which did not correlate with our study. ${ }^{4}$ Skeletal involvement of the tumor correlates with the literature studies. Yazawa et al in his study found axial skeleton was more commonly than the appendicular skeleton which correlates with our study were $75 \%$ involves the axial skeleton and $30 \%$ involves the appendicular skeleton. ${ }^{1}$ Commonest site of bone involved are the long bones and vertebral column. In our study the vertebral column and the femur are the most common bones involved which correlates with the literature studies. Metastasis to small bones are found to be very rare which correlated with study of brown et al.

Metastasis from epithelial origin is more common than mesenchymal origin and our study also shows similar findings. Out of 36 cases 34 cases where of epithelial origin which included adenocarcinomas from lung, prostate, colon, breast, thyroid, renal cell carcinoma, squamous cell carcinoma and 2 of them were of mesenchymalorigin which included leiomyosarcoma and myxoidfibrosarcoma. Asim et al and Vivek et al also found that metastasis of tumor from epithelial origin was more common than mesenchymal origin.

Immunohistochemistry plays a very important role in identifying the primary site of the tumor. A panel of markers were also done in our study to rule out the primary of the metastatic tumor. Table 1 shows the panel of IHC markers which are useful in determining the origin of tumor as epithelial, mesenchymal or lymphoid. Table 2 depicts the Specific markers and site of origin which helps us to determine the primary site. ${ }^{6}$ 
Table 1: Cell Lineage and IHC marker

\begin{tabular}{|l|c|c|}
\hline S. No & Cell lineage & Marker \\
\hline 1. & Epithelium & Pancytokeratin, EMA \\
\hline 2. & Smooth muscle & Actin \\
\hline 3. & Glial tissue & GFAP \\
\hline 4. & Lymphoid cell & CD45 \\
\hline 5. & Nerve & S 100 \\
\hline 6. & Skeletal muscle & Desmin, Myogenin \\
\hline 7. & Mesothelial cell & WT1, Calretenin \\
\hline 8. & Melanocyte & S100, HMB45 \\
\hline
\end{tabular}

Table 2: Specific IHC Marker and its tissue of origin

\begin{tabular}{|l|c|c|}
\hline S. No & Tumor site & Marker \\
\hline 1. & Breast & $\begin{array}{c}\text { GCDFP, ER, PR, E- } \\
\text { Cadherin }\end{array}$ \\
\hline 2. & Lung & $\begin{array}{c}\text { TTF-1, Surfactant protein, } \\
\text { napsin }\end{array}$ \\
\hline 3. & Ovary & WT1,S100,ER \\
\hline 4. & RCC & $\begin{array}{c}\text { CD10, RCC Marker, PAX } \\
\text { 2, PAX 8 }\end{array}$ \\
\hline 5. & Thyroid & TTF-1, Thyroglobulin, \\
\hline 6. & Prostate & PSA \\
\hline 7. & SCC & P63 \\
\hline 8. & Lymphoma & CD 45 \\
\hline 9. & Colon & CDX2, CK20+ \\
\hline
\end{tabular}

IHC is a very important ancillary technique that helps in prompt diagnosis. Clinical and radiological data also plays an important role in identifying the nature of tumor. Certain tumors are osteolytic, osteoblastic/Sclerotic and mixed. ${ }^{2}$ Table 3 shows the different tumors and their nature of appearance in radiology which helps us to identify the origin.

Table 3:

\begin{tabular}{|l|c|c|}
\hline S. No & Nature of lesion & Tumor \\
\hline 1. & Osteolytic & $\begin{array}{c}\text { Lung, Thyroid, } \\
\text { RCC, G.I.T, HCC }\end{array}$ \\
\hline 2. & Sclerotic & $\begin{array}{c}\text { Prostate, Breast, } \\
\text { Carcinoid, } \\
\text { Lymphoma }\end{array}$ \\
\hline 3. & Mixed & $\begin{array}{c}\text { Breast, Lung, } \\
\text { Cervix, Testicular, } \\
\text { Prostate }\end{array}$ \\
\hline
\end{tabular}

Hence, correlation of clinical, radiological and pathological studies helps us to detect the nature and origin of tumor which is also proven by other studies in

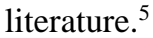

\section{Conclusion}

From the present study axial skeleton is more common site of metastasis as compared to appendicular skeleton. Long bones and vertebral column being the commonest sites for tumor deposit than the rest of the bones. Metastatic deposit from epithelial tumor are more common than the tumors of mesenchymal origin of which lung and prostate are the more common organs which tends to metastasize to the bone as seen in our study.

Statistical studies have shown that patients presenting with metastatic bone disease is increasing day by day but advancement in treatment and awareness among patients have helped in early detection and prompt treatment and longer survival.

\section{References}

1. Mandeep S Virk, Jay R Lieberman. Tumor metastasis to bone. Arthritis-research.com/content/9/S1/S5 2007.

2. Vivek Ajit Singh, Amber Haseeb, Alla Allden H Ali Alkubaisi. Incidence and outcome of bone metastatic disease at university Malaya Medical Centre. Singapore Med J 2014;55(10):539-546.

3. Asim Qureshi, Usman Shams, Azra Akhter, Sabiha Riaz. Metastatic bone disease as seen in our clinical practice Experience at a Tertiary Care Center in Pakistan. Asian Pacific J Cancer Prev, 13 (9), 4369-4371.

4. David J. Jacofsky, Deborah A. Frassica, Frank J Frassica. Metastatic bone disease. Clinical Review article. Hospital Physician November 2004.

5. D Ghartimangar, A Ghosh, R Narasimhan, OP Talwar. Patterns of hematological and non-hematological malignancies in bone marrow in a tertiary care hospital in Nepal 11 years study. Nepal Med Coll J 2012;14(3):187192.

6. SPARRC Postgraduate Handbook of Pathology 2017. Chapter 29 Immunohistochemistry Pg 363.

How to cite this article: Subalakshmi B, Nellaiyappan B, Thanka J. Analysis of metastatic bone disease in atertiary care centre. Ind J Pathol Oncol, 2018;5(3):505-508. 